\title{
Description d'un nouveau genre et de deux nouvelles espèces d'Ascalaphes afrotropicaux (Neuroptera, Ascalaphidae)
}

\section{Bruno Michel}

\section{Abstract}

Description of a new genus and two new species of afrotropical Ascalaphidae (Neuroptera). The genus Farakosius $\mathrm{n}$. gen. and the species $F$. chloeae n. sp. and F. thaisae n. sp. are described.

\section{Résumé}

Le genre Farakosius n. gen. et les espèces $F$. chloeae n. sp. et $F$. thaisae n. sp. sont décrits du Mali.

\section{Citer ce document / Cite this document :}

Michel Bruno. Description d'un nouveau genre et de deux nouvelles espèces d'Ascalaphes afrotropicaux (Neuroptera, Ascalaphidae). In: Bulletin de la Société entomologique de France, volume 103 (1), mars 1998. pp. 45-50; https://www.persee.fr/doc/bsef_0037-928x_1998_num_103_1_17392

\section{Ressources associées :}

Neuroptera

Ascalaphidae

Fichier pdf généré le 24/09/2019 


\title{
Description d'un nouveau genre et de deux nouvelles espèces d'Ascalaphes afrotropicaux (Neuroptera, Ascalaphidae)
}

\author{
par Bruno MICHEL \\ CIRAD/IER Centre Régional de Recherche Agronomique, B.P. 16, Sikasso, Mali
}

\begin{abstract}
Résumé. - Lc genre Farakosius n. gen. et les espèces $F$. chloeae n. sp. et $F$. thaisae n. sp. sont décrits du Mali. Summary. - Description of a new genus and two new species of afrotropical Ascalaphidae (Neuroptera). The genus Farakosius n. gen. and the species $F$. chloeae n. sp. and $F$. thaisae n. sp. are described.

Mots clés. - Neuroptera, Ascalaphidae, Farakosius n. gen., n. spp., région afrotropicale, Mali.
\end{abstract}

La nomenclature utilisée est celle proposée par TJEDER (1977, 1992).

\section{Diagnose du genre Farakosius n. gen.}

Espèce-type du genre: Farakosius chloeae n. sp.

Par la forme des ectoproctes, ce genre appartient à la tribu des Ascalaphini telle qu'elle est définie par TJEDER \& HANSSON (1992). La forme du gonarcus et des paramères rappelle celle du genre Mabiza Tjeder \& Hansson, 1992, mais la présence d'une fossette sensorielle sur les palpes labiaux et d'une touffe de soies sur les joues l'en séparent nettement. En utilisant la clé des genres d'Ascalaphini afrotropicaux publiée par ces auteurs, on aboutit au genre Brevibarbis Tjeder \& Hansson, 1992, qui, entre autres caractères distinctifs, présente les joues densément pileuses et le complexe gonarcus-paramc̀rcs de forme différente.

Pour inclure le genre Farakosius n. gen., la clé des genres d'Ascalaphini afrotropicaux publiée par TJEDER \& HANSSON (1992) doit être modifiée comme suit :

1. Genae hairy 2

- Genae hairless 6 (remplace 5)

2. Genae with just a little tuft of hairs in the lower part Farakosius n. gen.

- Genae with dense hairs 3

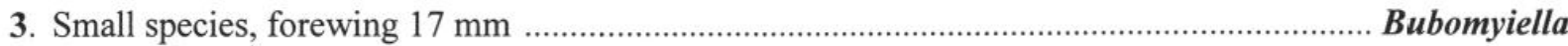

- Larger species, forewing 20-29 mm Bubomyiella

4. Hindwing partly to completely infumate, forewing hyaline; vertex wide, about $1.9 \mathrm{x}$ as wide as width of an eye in dorsal view Encyopsidius

- Both pairs of wings hyaline or with membrane near crossveins shaded with grey; vertex narrower ...... $\mathbf{5}$

5. Wingveins hairless; several crossveins shaded with grey, especially in distal part of wings ...... Strixomyia

- Wingveins with very short hairs; wing membrane unspotted ................................................. Brevibarbis 6......

Tête. - Plus large que le thorax. Vertex nettement plus étroit que la largeur d'un ceil en vue dorsale, avec une touffe de soies dorsales dressées. Front garni de soies formant des touffes le long des côtés et du bord inférieur. Clypéus avec des soies éparses. Marge inférieurc du labre bordéc par des soies dorées clairsemées, plus raides. Joues avec une petite touffe de soies peu denses dans le 1/4 inférieur, le long de la marge interne (fig. 1). Pleurostomes présents. Sclérites post-oculaires, face postérieure du vertex et occiput inermes. Antennes plus courtes que la distance entre la base des ailes antérieures et le ptérostigma. Scape plus large que haut avec le disque recouvert de longues soies dressées. Trois ou quatre premiers flagellomères avec quelques soies apicales couchées sur le segment suivant. Massues arrondies recouvertes de très courtes soies couchées. Partie inférieure des yeux moins développée que la supérieure. Palpes labiaux avec une fossette sensorielle sur la face supérieure (fig. 1). 
Thorax. - Recouvert de soies dressées assez denses. Face supérieure des tibias antérieurs avec de courtes soies formant un revêtement en forme de brosse. Pattes antérieures et intermédiaires avec les éperons sub-égaux aux deux premiers articles des tarses. Quatre premiers tarsomères égaux, le cinquième égal aux quatre premiers réunis. Pattes postérieures avec les éperons égaux au premier tarsomère qui est environ une fois et demie plus long que le deuxième. Face inférieure des tarsomères avec des soies en forme d'épines plus longues dans les angles apicaux. Ailes semblables dans les deux sexes avec l'angle anal arrondi. Ailes antérieures plus longues que les postérieures. Réticulation dense avec des soies en forme d'épines sur les nervures. Ptérostigma présent avec 2 à 3 nervures. Membrane inerme. Aire apicale avec 2 rangées de cellules, trois chez quelques rares individus. $\mathrm{M}$ et $\mathrm{Cul}$ assez proches, parallèles et régulièrement courbées vers le bord postérieur des ailes. Aux ailes antérieures, Cu2 et $1 \mathrm{~A}$ fusionnées légèrement après l'angle anal; 4 à 6 nervures avant Rs. Aux ailes postérieures, 3 à 4 nervures avant Rs.

Abdomen. - Plus court que les ailes postérieures dans les deux sexes. Tergite 1 avec 2 touffes latérales de soies dressées. Tergite 2 avec des soies longues, en particulier le long des marges latérales. Autres tergites avec des très courtes soies éparses en forme d'épine. Tergites 3 à 7 avec une entaille latérale (fig. 6). Sternite 1 bombé. Sternite 2 avec de longues soies dressées, les suivants avec des soies beaucoup plus courtes. Ectoproctes des mâles en forme d'écailles convexes sans prolongements latéraux ni expansions. Gonarcus et paramères voir figures 8 à 10 et 13 à 15 . Pelta présente, de forme allongée. Chez les femelles (fig. 11 et 16) ventrovalves et distivalves bien développées. Interdens présente. Linguella non divisée. Ectoproctes en forme d'écailles convexes.

Etymologie. - formé à partir du nom de la localité-type Farako.

\section{Farakosius chloeae n. sp.}

HOLOTYPE (fig. 2): $\delta^{\star}$, Mali, chutes de Farako $\left(11^{\circ} 12^{\prime} \mathrm{N}-05^{\circ} 29^{\prime} \mathrm{W}\right.$ ), prairie à graminées en bordure de forêt galerie, 4.VIII.1996 (B. Michel). ALLOTYPE (fig. 3): $\$$, idem, 6.VIII.1996 vers $17 \mathrm{~h}(\mathrm{~B}$. Michel). PARATYPES, 6 o, 14 : idem, $2 \%, 6$. VIII.1996 entre 10 et $12 \mathrm{~h} ; 4 q$, 6.VIII.1996 vers $17 \mathrm{~h} ; 1$ t, 29 , 8.VIII.1996 vers $17 \mathrm{~h} ; 5 \delta, 3 q, 21$.VIII. 1997 entre $15 \mathrm{~h} 30$ et 17 h; $19,29$. VIII.1996; $19,3 . X .1996 ; 19,6 . X I .1996$ vers 16 h 30. Tous in coll. Michel.

Dimensions. Mâles: ailes antérieures $28 \mathrm{~mm}$; ailes postérieures $24 \mathrm{~mm}$; abdomen $18 \mathrm{~mm}$. Femelles : ailes antérieures $26-35 \mathrm{~mm}$; ailes postérieures $22-30 \mathrm{~mm}$; abdomen $15-20 \mathrm{~mm}$.

Coloration générale roux clair, avec les dessins noirs et la bande thoracique jaune bien visibles. Extrémité de l'abdomen du mâle blanche.

Tête. - Vertex roux avec les soies grises associées à des soies noires sur les côtés. Face (fig. 1) jaune avec les joues et les pleurostomes plus clairs. Soies blanches. Sclérites post-oculaires jaune clair. Occiput couleur ivoire avec une grande tache brune présentant une encoche dans la moitié supérieure. Scape jaune clair avec des soies grises et noires. Pédicelle plus sombre avec les soies noires. Premier flagellomère avec des soies noires absentes antérieurement. Articles 2 à 4 avec 1 à 2 soies noires latérales implantées sur le bord apical. Autres articles inermes avec la face ventrale noire et la face dorsale couleur ivoire. Massues noires recouverte d'une courte pilosité noire. Pièces buccales jaune clair avec l'apex des derniers articles des palpes assombri. Mandibules blanc ivoire avec l'extrémité brune devenant noire à l'apex.

Thorax. - Face dorsale: pronotum jaune avec les côtés et deux bandes dorsales noires; mésoprescutum jaune avec deux bandes dorsales noires continuant celles du pronotum; méso-scutum roux avec une bande centrale jaune; méso-scutellum jaune avec deux taches latérales noires en forme de croissant; méso-postnotum jaune avec deux bandes latérales noires; méta-scutum roux avec la partie antérieure jaune bordée de noir; méta-scutellum jaune bordé de noir. Côtés blancs à jaunes plus ou moins obscurcis vers la partie dorsale, avec quatre taches noires formant une ligne discontinue allant du bord antérieur du méso-anepisternum au bord antérieur du méta-anépisternum. Face ventrale noire.

Pattes. - Coxae noires. Fémurs et tibias jaunes avec une ligne longitudinale noire bien marquée sur les pattes intermédiaires, et à peine esquissée sur les fémurs postérieurs. Sur les tibias intermédiaires, cette ligne est bordée vers l'extérieur par deux taches noires allongées. Tarses jaunes, légèrement assombris en dessous. Griffes brunes plus ou moins foncées. 

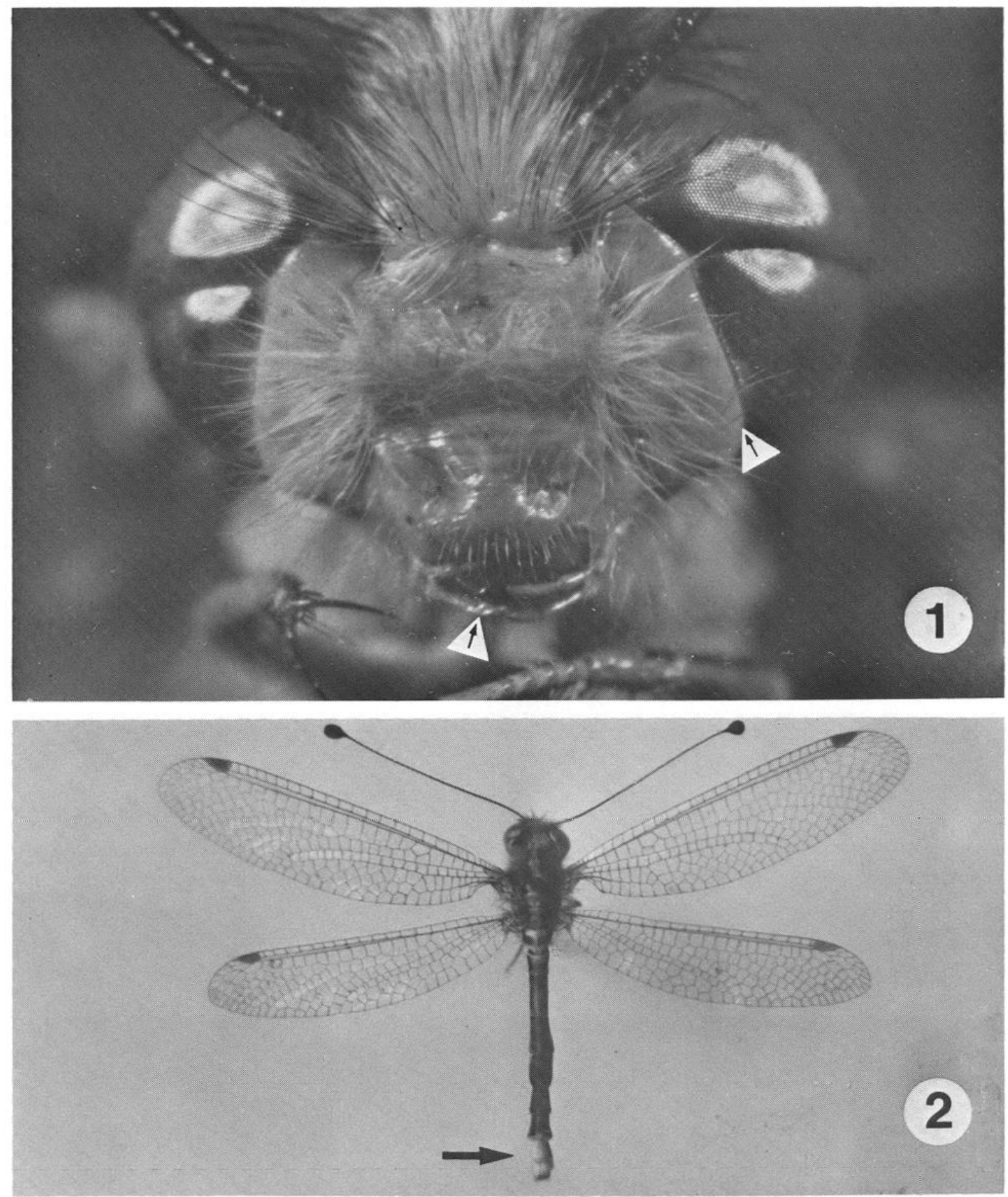

Fig. 1-2. - Farakosius chloeae n. gen., n. sp., holotype, ठ. - 1, tête vue de face (x 20). La touffe de soies jugale gauche et la fossette labiale droite sont visibles (flèches); -2 , habitus (x 1,6), noter la coloration blanche de l'extrémité abdominale (flèche).

Ailes. - Membrane hyaline à très légèrement enfumćc. Ncrvurcs longitudinales orangées, les tranversales noires.

Abdomen. - Mâle: tergites 1 à 7 roux bordés latéralement d'un liséré noir élargi le long des marges antérieures et postérieures (fig. 6). Tergites 8 et 9 et ectoproctes blancs (fig. 7). Sternites blancs avec un trait noir longitudinal médian plus ou moins net, formant une ligne discontinue le long de l'abdomen. Femelle: même coloration générale. Les taches noires des tergites et des sternites bien marquées. Ventrovalves avec une tache noire peu étendue. Distivalves jaunes (fig. 11). Ectoproctes jaunes.

Etymologie : dérivé du prénom Chloé. 

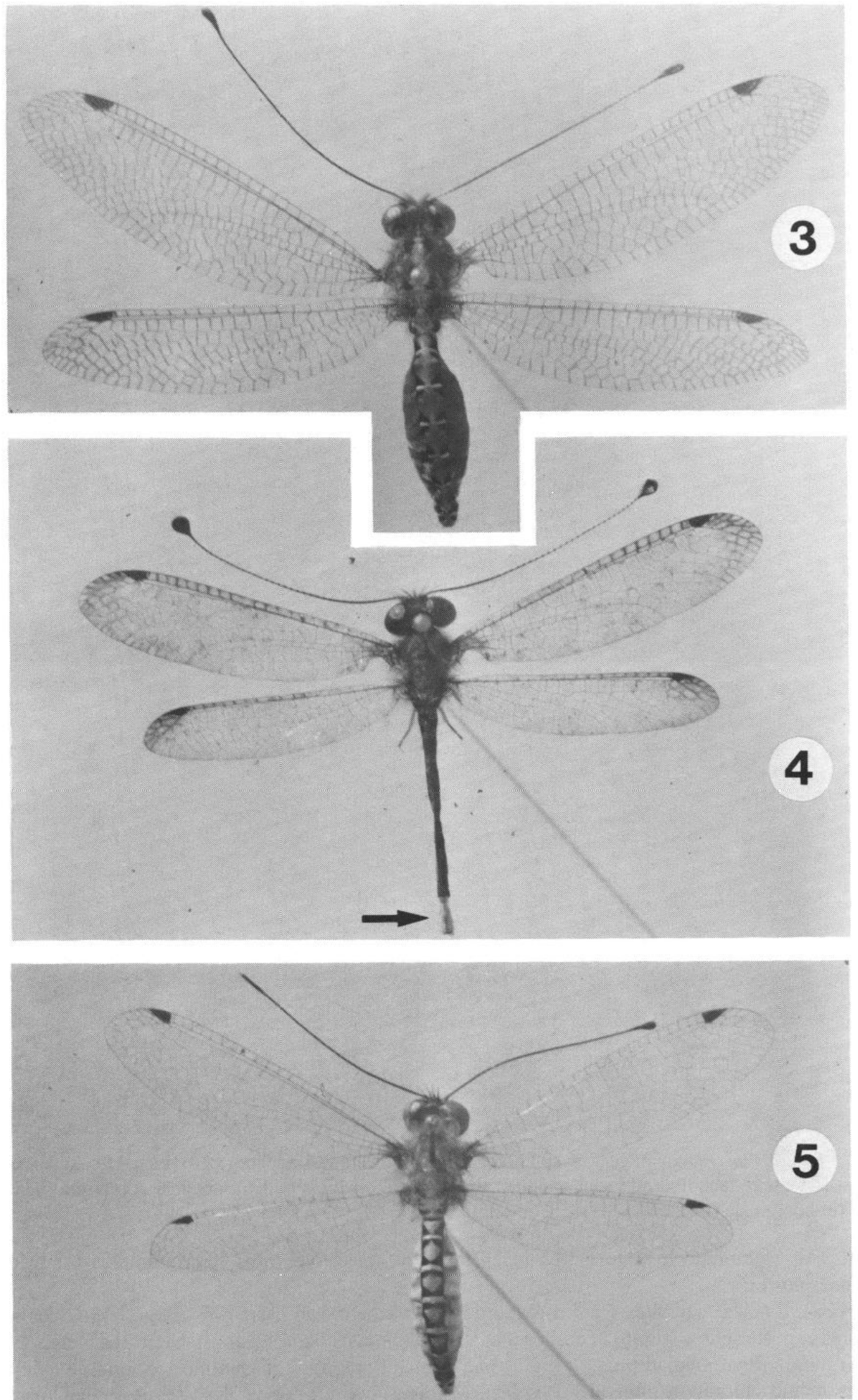

Fig. 3-5. - 3, Farakosius chloeae n. gen., n. sp., allotype, $\$(\times 1,8) .-4$, Farakosius thaisae n. gen., n. sp., holotype, $\delta(x 1,8)$, noter la coloration blanche de l'extrémité abdominale (flèche). -5 , Farakosius thaisae $\mathrm{n}$. gen., n. sp., allotype, $\$$ ( $x$ 1,7). 


\section{Farakosius thaisae n. sp.}

HOLOTYPE (fig. 4): $\delta$, Mali, chutes de Farako, prairie à graminées en bordure de forêt galerie, 28.X.1996 vers $16 \mathrm{~h}$ (B. Michel). ALLOTYPE (fig. 5): + , idem, 6.XII.1996 vers $16 \mathrm{~h}$ (B. Michel). PARATYPES, $100^{\circ}, 20 \%$ : idem, 20, $11 \%, 6 . X \mathrm{X} .1996$ entre $16 \mathrm{~h} 30$ et $17 \mathrm{~h} ; 50^{*}, 19$, 15.XI.1995 vers $11 \mathrm{~h} ; 3 \%, 17 . X I .1995 ; 2 q, 4 . X I I .1996 ; 2 \delta, 1 \%, 6 . X I I .1996$ entre 9 et $10 \mathrm{~h}$; $10,2+, 8 . X I I .1995$ vers $11 \mathrm{~h}$. Tous in coll. Michel.

Dimensions (en mm). - Mâles : ailes antérieures 22-25; ailes postérieures 18-21; abdomen 15-16. Femelles: ailes antérieures 22-27; ailes postérieures 18-22; abdomen 15-16.

Mâle plus sombre que chez l'espèce précédente, avec les dessins noirs moins nets. Extrémité de l'abdomen également blanche. Femelle plus claire, avec les taches jaunes et noires bien nettes. Ailes hyalines. Taille inférieure.

Tête. - Vertex roux clair avec des soies gris foncé à noires. Face jaune avec les joues plus ou moins éclaircies, parfois blanches. Soies blanches; celles qui bordent le labre plus courtes et dorées. Sclérite post-oculaire jaune clair à blanc ivoire. Occiput jaune avec une tache brune allongée transverse dans le 1/4 supérieur et une tache noire d'extension variable de chaque côté du cou. Scape jaune avec des soies blanches à grises. Pédicelle et 4 ou 5 premiers flagellomères bruns. Les suivants noirs chez la femelle, et avec la face dorsale jaune chez le mâle à partir du $13^{\mathrm{e}}$ ou $14^{\mathrm{e}}$. Massue antennaire arrondie brune, avec chez le mâle la face dorsale des 2 ou 3 premiers articles éclaircie. Mandibules jaunes avec la partie distale brune devenant noire à l'extrémité. Autres pièces buccales jaunes avec l'extrémité des derniers articles des palpes plus ou moins sombres.

Thorax. - Face dorsale rousse chez le mâle, jaune chez la femelle, avec les différentes parties bordées de noir; ces dessins noirs bien délimités chez les femelles et plus ou moins nets chez les mâles. Soies brunes sauf le long du bord postérieur du méso-scutellum et sur le méta-scutellum où elles sont gris argenté. Côtés du thorax avec la moitié supérieure noire et la moitié inférieure jaune devenant blanche postérieurement chez les femelles. Soies grises. Face sternale noire avec des soies grises.

Pattes. - Coxae noires, les autres articles jaunes. Fémurs antérieurs et intermédiaires avec des soies grises fines et des soies plus robustes noires plus nombreuses sur les fémurs intermédiaires. Fémurs postérieurs avec uniquement des soies grises fines. Tibias avec des soies robustes noires. Griffes brunes.

Ailes. - Membrane hyaline chez les femelles, parfois très légèrement teintée de jaune; hyaline à brune chez les mâles. Nervures longitudinales jaune plus ou moins foncé chez les femelles, brunes chez les mâles. Nervures transversales et secteur radial jaunes chez les femelles, noirs chez les mâles.

Abdomen. - Mâle : face dorsale rousse avec les tergites 1 à 7 bordés latéralement de noir. Marge postérieure du tergite 7, tergites 8,9 et ectoproctes blancs (fig. 12). Face ventrale jaune avec sur chaque sternite une ligne longitudinale centrale noire bien nette, s'élargissant à l'extrémité, et formant une ligne continue au moins sur les trois premiers segments. La partie apicale des lignes des deux derniers sternites atteint les marges latérales. Plaque sous-génitale avec un liséré médian et les côtés noirs. Femelle: face dorsale noire avec sur les tergites 1 à 8 une grande tache jaune discale et un épais liseret blanc le long du bord postérieur. $9^{\mathrm{e}}$ tergite avec 2 taches latérales noires. Ectoproctes jaunes. Face ventrale jaune avec les mêmes dessins que chez le mâle, mais plus épais. Ventrovalves en grande partie noires. Distivalves avec une tache noire au contour irrégulier (fig. 16).

Etymologie: dérivé du prénom Thaïs.

\section{Ecologie}

Les deux espèces ont été capturées dans une savane à graminées située en bordure d'une forêt galerie et dont le sol est sablonneux. Il s'agit d'une station relativement plus humide que les prairies du type "bowal" rencontrées communément dans la région de Sikasso (sud-est du Mali) sur des sols constitués essentiellement par la cuirasse ferrugineuse. En début de végétation, les adultes se réfugient dans le tapis végétal d'une hauteur de 15 à $30 \mathrm{~cm}$, constitué en majeure partie par le feuillage des graminées. Par la suite, lorsque les plantes se sont développées, ils se posent sur les tiges des graminées à une hauteur d'environ $1 \mathrm{~m}$. Les deux espèces sont facilement repérables grâce à la coloration blanche de l'extrémité de l'abdomen. D'après nos observations, elles volent préférentiellement en fin de matinée et en fin d'après-midi entre $16 \mathrm{~h}$ et $18 \mathrm{~h}$ environ. 
(6
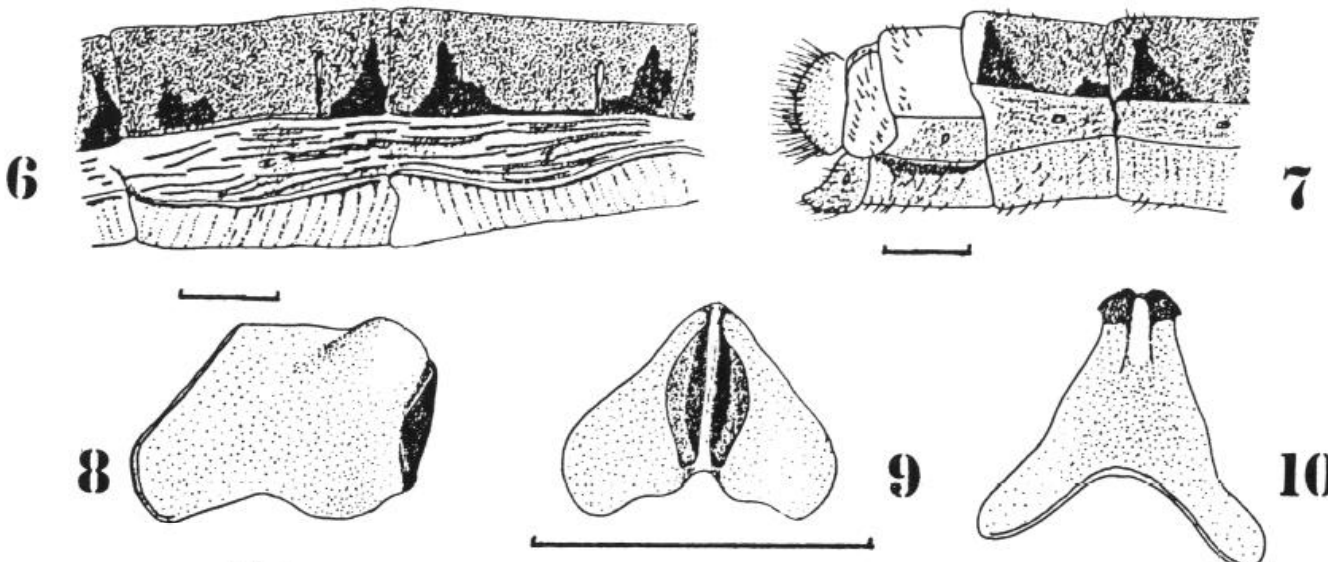

()
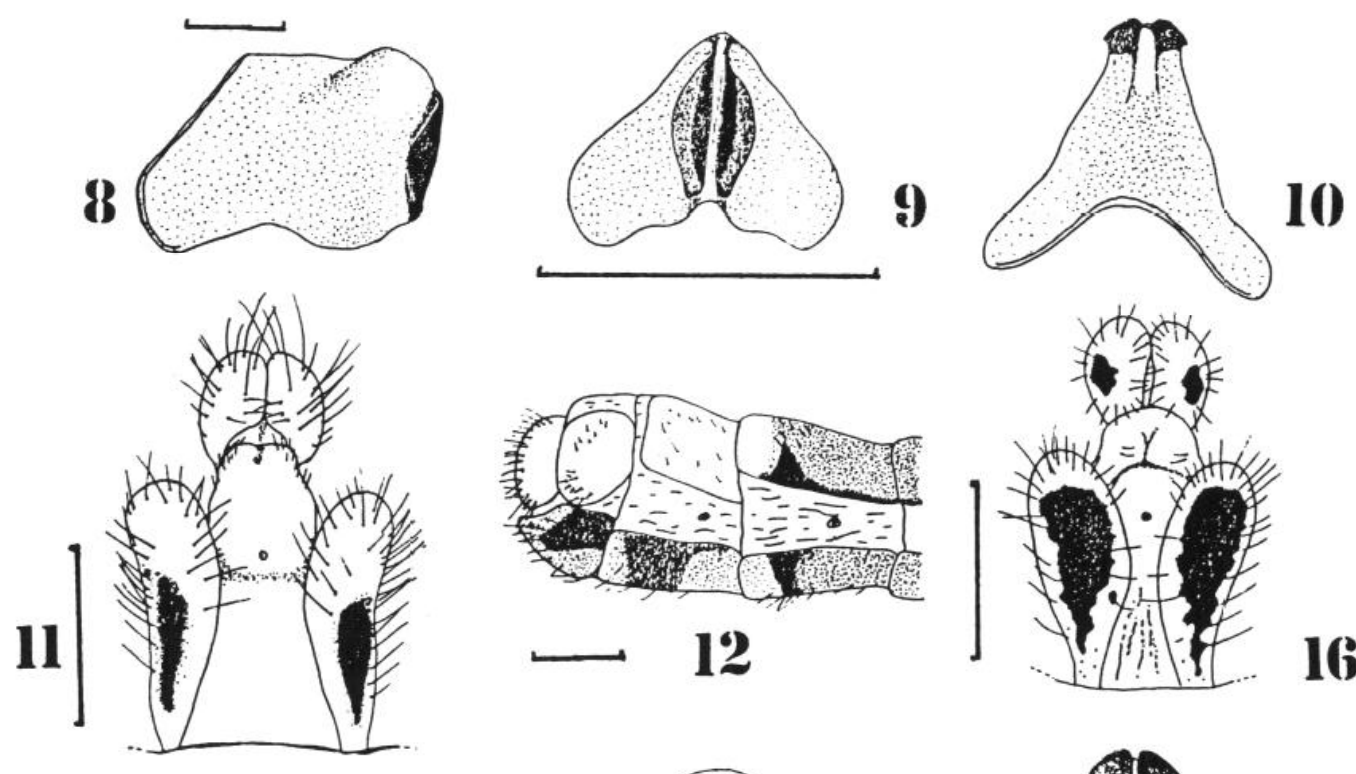

16
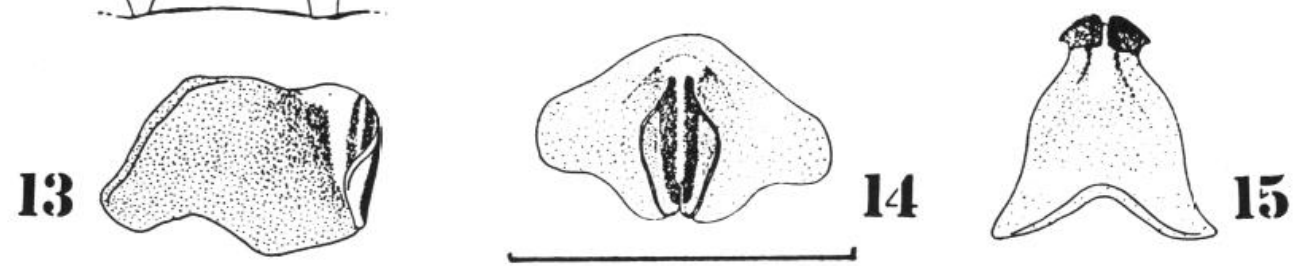

Fig. 6-16. -6 à 11, Farakosius chloeae n. gen., n. sp. $-6, \delta$ : segments abdominaux IV et $\mathrm{V}$ en vue latérale droite. $-7, \delta$ : extrémité abdominale. -8 , gonarcus-paramères en vue latérale gauche. -9 , id., en vue postérieure. $-10, i d$., en vue dorsale. $-11, q$ : ventrovalves, interdens, linguella et distivalves en vue ventrale. - 12 à 16, Farakosius thaisae n. gen., n. sp. $-12, \delta^{\dagger}$ : extrémité abdominale. - 13, gonarcus-paramères en vue latérale gauche. $-14, i d$, en vue postérieure. $-15, i d$., en vue dorsale. $-16, \%$ : ventrovalves, interdens, linguella et distivalves en vue ventrale. (Echelles $=1 \mathrm{~mm}$ ).

$F$. chloeae a été capturé du 4.VIII au 6.XI. C'est donc une espèce qui vole pendant la saison des pluies et en début de saison sèche. $F$. thaisae apparaît plus tardivement. Les dates de captures, qui vont du 28.X au 8.XII, indiquent que les adultes ne commencent à voler qu'à la fin de la saison des pluies, et qu'ils sont par conséquent plus nettement inféodés à la saison sèche.

REMERCIEMENTS. - Je remercie M. C. Hansson, de l'Université de Lund (Suède), pour son aimable collaboration.

\section{AUTEURS CITÉS}

TJEDER B., 1977. - Distal abdominal segments and sclerotized parts of genitalia in Ascalaphidae (Neuroptera). Annales Entomologica Fennici, 43 (2): 61-65.

1992. - The Ascalaphidae of the Afrotropical Region (Neuroptera). 1. External morphology and bionomics of the family Ascalaphidae, and taxonomy of the subfamily Haplogleniinae including the tribes Proctolyrini n. tribe, Melambrotini n. tribe, Campylophlebini n. tribe, Tmesibasini n. tribe, Allocormodini n. tribe, and Ululomyiini n. tribe of Ascalaphinae. Entomologica Scandinavica, suppl. $41: 1-169$.

TJEDER B. \& HANSSON C., 1992. - The Ascalaphidae of the Afrotropical Region (Neuroptera). 2. Revision of the tribe Ascalaphini (subfam. Ascalaphinae) excluding the genus Ascalaphus Fabricius. Entomologica Scandinavica, suppl. 41 : 170-237. 